\title{
Determining the immunohistochemical expression of GLUT1 in renal cell carcinoma using the HSCORE method
}

\author{
MARIN OGOREVC ${ }^{1 *}$, ANTE STRIKIC $^{2 *}$ and SANDRA ZEKIC TOMAS ${ }^{3}$ \\ ${ }^{1}$ School of Medicine, University of Split; ${ }^{2}$ Department of Oncology, University Hospital Split; \\ ${ }^{3}$ Institute for Pathology, University Hospital Split, 21000 Split, Croatia
}

Received April 26, 2021; Accepted July 6, 2021

DOI: $10.3892 /$ br.2021.1455

\begin{abstract}
The aim of the present study was to compare the immunohistochemical expression of glucose transporter 1 (GLUT1) between the most common histological types of renal cell carcinoma (RCC), and to determine whether a correlation between GLUT1 expression and nuclear grade or tumor size exists. A total of 19 RCC samples were selected for the study, consisting of 8 clear cell (cc)RCC and 11 non-ccRCC tissues. Immunohistochemistry for GLUT1 was performed on formalin-fixed and paraffin-embedded sections using GLUT1 antibodies. All data analyses were performed using the MedCalc software. There was a higher immunohistochemical expression of GLUT1 in the ccRCC group compared with the non-cc group, but there was no difference in GLUT1 expression between groups of RCCs with differing nuclear grades. No significant correlation between GLUT1 expression and tumor size was found. The higher immunohistochemical expression of GLUT1 in ccRCC may be a contributing factor to the clinical characteristics and behavior of that group of carcinomas. These results suggest that GLUT1 expression cannot be used as a prognostic factor for RCC, but it may be used as a predictive factor in the future.
\end{abstract}

\section{Introduction}

Renal cell carcinoma (RCC) accounts for $2.2 \%$ of all cancer diagnoses worldwide. It is the 7th most common type of cancer

Correspondence to: Dr Sandra Zekic Tomas, Institute for Pathology, University Hospital Split, Spinčićeva 1, 21000 Split, Croatia

E-mail: szekic@mefst.hr

${ }^{*}$ Contributed equally

Abbreviations: RCC, renal cell carcinoma; ccRCC, clear cell RCC; pRCC, papillary RCC; chRCC, chromophobe RCC; VHL, Von Hippel-Lindau; HIF, hypoxia-inducible factor; GLUT1, glucose transporter 1

Key words: kidney neoplasms, renal cell carcinoma/metabolism, GLUT1, immunohistochemistry, glucose/metabolism in developed countries, with the highest incidence in North America, followed by Western Europe (1). RCC is responsible for $1.8 \%$ of global cancer deaths and $2.4 \%$ of cancer-related deaths in the US (2). The 5-year survival rate for all individuals with RCC is $76 \%$, making it one of the deadliest urological cancers (1). RCC is routinely diagnosed by pathohistological analysis using the protocol devised by the College of American Pathologists, and histological type is determined based on the 2016 WHO classification of tumors of the urinary system $(3,4)$. Localized RCCs are treated by surgical resection, whereas metastatic RCCs are treated by targeted therapy according to the National Comprehensive Cancer Network guidelines (5). The most common histological type is clear cell RCC (ccRCC), which constitutes 70-90\% of all RCCs. Papillary RCC (pRCC) and chromophobe chRCC (chRCC) are the next most common types, constituting $10-15 \%$ and $3-5 \%$ of all RCC cases, respectively (6). ccRCC has a worse prognosis and is more likely to be diagnosed in the first instance at a higher stage than pRCC or chRCC (7). Prior research found that $90 \%$ of ccRCCs have mutations present in the von Hippel-Lindau tumor suppressor (VHL) gene (8). The VHL protein has a role in the degradation of hypoxia-inducible factors (HIFs) and in its absence, HIFs become stabilized $(9,10)$. Stabilized HIFs act as transcription factors and bind to hypoxia response elements on target genes, upregulating the expression of proteins involved in metabolic reprogramming, induction of angiogenesis, as well as other oncogenic processes (11). HIFs affect cell metabolism primarily by increasing the expression of glucose transporter 1 (GLUT1) protein (9). GLUT1 is one of 14 members of a family of integral membrane proteins whose purpose is the transport of monosaccharides (primarily glucose) and other small carbon compounds across the cell membrane (12). Cancer cells depend on glucose metabolism for energy production and the rate-limiting step of glucose metabolism is uptake across the membrane $(13,14)$. Thus, it comes as no surprise that expression of glucose transporters, such as GLUT1, is upregulated in several types of cancer cells (15). GLUT1 expression is positively correlated with tumor metabolic activity in several types of cancer, including colorectal cancer, ovarian cancer and melanoma (16-18). High expression of GLUT1 has also been used as a prognostic biomarker, indicating poor survival, in several types of cancer, such as colorectal, ovarian, bladder and esophageal carcinoma (19-23). In addition, GLUT proteins have been suggested as targets for cancer therapy; it has been 
shown that inhibition of glucose uptake can cause metabolic stress and activate tumor suppressor pathways in cells $(24,25)$. From the GLUT family, GLUT1 is the most extensively researched member, and anti-GLUT1 therapy exhibits induction of growth inhibition and/or cell death in several cancer cell types (26-29). Glucose uptake inhibitors can also sensitize cancer cells to cytotoxic therapy, suggesting the possibility of its use as an adjuvant $(13,30)$.

The aim of the present study was to determine the correlation between GLUT1 expression and the clinical characteristics of RCCs, specifically histological type, nuclear grade and tumor size.

\section{Materials and methods}

Tissue samples. The present study was performed at the Institute of Pathology, Forensic Medicine and Cytology, Split University Hospital Centre (Split, Croatia). The present study was approved by the Hospital Ethics Committee of the University Hospital Centre in Split, Croatia (approval no. 2181-147-01/06/M.S.-20-9), and was performed in accordance with the ethical standards described in the 1964 Declaration of Helsinki and its later amendments (31). Consent from patients was not required as the study was retrospective, and patients had provided consent for use of their data/samples during their visits to the hospital. Specifically, considering that the hospital is a university hospital, all patients were informed prior to surgical and/or diagnostic procedures that the material obtained during these procedures may be used for education and/or research purposes, and they signed consent forms for these procedures and the use of their data/materials.

The institute's database of pathohistological reports was searched to select appropriate RCC samples for the study. Samples were taken from the institute's archive; all samples were initially obtained from patients between January 2015 and December 2018. All chosen samples were from primary kidney tumors of adults with only one tumor focus present at the time of nephrectomy. To avoid confounding factors, samples were chosen in such a manner that there was no large difference between the sex and age of patients with RCC. Additionally, the samples that were chosen had no marked lymphovascular invasion, necrosis, sarcomatoid and/or rhabdoid features present, to make the samples as uniform as possible and to assess the immunohistochemical expression more accurately. Additionally, samples where the tumor was limited to the kidney were used to obtain precise measurements of tumor size. Overall, 19 paraffin blocks containing formalin-fixed RCC samples were collected from the institute's archive, 8 of those being ccRCC (median age 58.5, age range 49-86, 4 males and 4 females), 7 pRCC (median age 80, age range 56-83, 5 males and 2 females) and 4 chRCC (median age 54, age range 44-67, 3 males and 1 female). Out of the pRCC cases, 4 were type I and 3 were type II.

RCC tissue preparation and immunohistochemistry. From each paraffin block, a $4 \mu \mathrm{m}$-thick RCC section was cut, mounted and dried at $37^{\circ} \mathrm{C}$. Subsequently, sections were stained with hematoxylin and eosin using an Automatic Stainer HE600, according to the manufacturer's protocol (VentanaRoche; Roche Diagnostics $\mathrm{GmbH}$ ). All the sections were re-examined by a pathologist according to the standard protocol described by the College of American Pathologists (3). The histopathological analysis was performed manually using a light microscope (Olympus BX41; Olympus Corporation) at x400 magnification, and Cell D1 Image analysis software version Cellsense (Olympus Corporation). Immunostaining was performed on the same serial section of each RCC sample, as follows: Paraffin sections were mounted on super frost slides (Thermo Fisher Scientific, Inc.) and processed in an automatic stainer (Ventana Bench Mark Ultra Autostainer; VentanaRoche; Roche Diagnostics $\mathrm{GmbH}$ ). For the detection of GLUT1-positive RCC cells, primary 'ready-to-use' monoclonal mouse antibodies (RRID:AB_10578246; cat. no. SPM498; 1:100; Novus Biologicals, Ltd.) were applied (Fig. 1). The UltraView Universal DAB Detection kit (RRID:AB_2753116; cat. no. G32061; VentanaRoche; Roche Diagnostics GmbH) was used as the secondary antibody (supplied ready to use). Brown staining of the cell membrane and/or cytoplasm was considered positive. Erythrocytes present in blood vessels inside the RCC tissue were used as a positive control. The expression of GLUT1 in the samples was determined by the HSCORE method using the equation: HSCORE $=\sum \mathrm{P} i$ $(i+1)$, where $i=$ intensity of staining with a value of 1 (weak), 2 (moderate), or 3 (strong), and $\mathrm{P} i$ is the percentage of stained RCC cells of each intensity (32). For every RCC sample, 10 representative fields of view were chosen and an HSCORE was calculated for each of them. The HSCORE of the entire sample was the arithmetic mean of HSCOREs of the 10 individual fields of view.

Statistical analysis. The normality of distributions was tested using a Shapiro-Wilk test. The mean and standard deviation were used as the measures of central tendency and variance for normally distributed data, whereas the median and interquartile range were used for data that was not normally distributed. A Fisher's exact test was used to determine the differences between the nominal characteristics of the groups of RCCs. A Student's t-test (unpaired) and ANOVA with Scheffé post-hoc test were performed to assess the differences in the age of the patients according to the type of RCC. A Mann-Whitney U and Kruskal-Wallis with a Conover post-hoc test was used to assess the differences in GLUT1 expression for groups without normal distributions, whereas an Independent sample t-test was used for groups that exhibited normal distribution. Pearson's correlation analysis was used to determine the correlation between tumor size and GLUT1 expression. All data were analyzed using MedCalc Statistical Software version 19.1.2 (MedCalc Software, Ostend, Belgium; medcalc. org; 2019, RRID:SCR_015044). P<0.05 was considered to indicate a statistically significant difference and all confidence intervals (CI) are stated at the $95 \%$ level.

\section{Results}

To compare the immunohistochemical expression of GLUT1 in different histological types of RCCs, the tissues were split into 2 groups: ccRCCs $(n=8)$ and non-ccRCCs $(n=11)$. There was no statistically significant difference in terms of age, sex or nuclear grade between the two groups. There was a statistically significant difference in tumor size measured by the greatest 


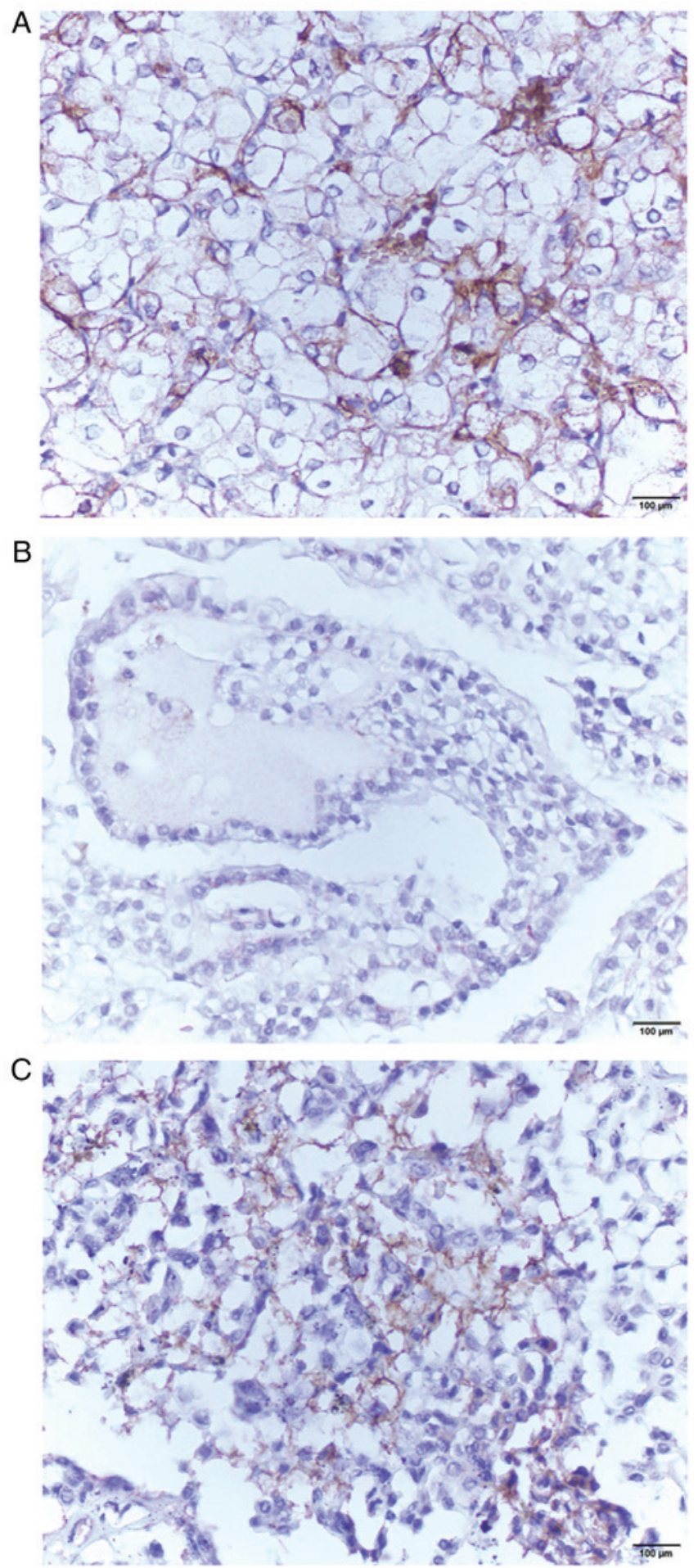

Figure 1. Light microscopy of RCC samples stained with anti-GLUT1 antibodies. (A) A moderate to strong, diffuse, membranous staining for GLUT1 can be observed in an example of clear cell RCC, (B) whilst only weak, focal, cytoplasmic staining can be observed in a papillary RCC sample, and (C) weak to moderate, cytoplasmic, with some foci of strong membranous staining in a chromophobe RCC sample. Strongly stained erythrocytes in spaces between RCC cells were used as an internal positive controls. Magnification, $\mathrm{x} 400$. Scale bar, $100 \mu \mathrm{m}$. RCC, renal cell carcinoma; GLUT1, glucose transporter 1 .

diameter of the tumor $(\mathrm{P}=0.038)$ between the two groups; the ccRCC group was larger in size (Table I). There was also a statistically significant difference in GLUT1 expression based on the HSCORE $(\mathrm{P}=0.044)$ between the two groups, with the
Table I. Characteristics of the patients with RCC.

\begin{tabular}{lccc}
\hline Characteristics & $\begin{array}{c}\text { Clear cell } \\
\text { RCC, } \mathrm{n}=8\end{array}$ & $\begin{array}{c}\text { Non-clear cell } \\
\text { RCC, } \mathrm{n}=11\end{array}$ & P-value \\
\hline $\begin{array}{l}\text { Age, years } \\
\text { Sex }\end{array}$ & $58.5(49-86)$ & $67(44-83)$ & 0.495 \\
Male & $4(21.1)$ & $8(42.1)$ & 0.324 \\
Female & $4(21.1)$ & $3(15.8)$ & \\
Grade $^{\mathrm{c}}, \mathrm{n}(\%)$ & & & \\
Low $^{\mathrm{c}}$ & $4(21.1)$ & $4(21.1)$ & 0.658 \\
High & $4(21.1)$ & $7(36.8)$ & \\
Tumor size & $7.31 \pm 3.32$ & $4.36 \pm 2.4$ & $0.038^{\mathrm{a}}$ \\
\hline
\end{tabular}

${ }^{\mathrm{a}} \mathrm{P}<0.05$. ' Independant samples t-test. ${ }^{\mathrm{c}}$ Fisher's exact two-sided test. ${ }^{\mathrm{d}}$ Median (age range) ${ }^{\mathrm{e}}$ Mean \pm standard deviation. RCC, renal cell carcinoma.

Table II. Comparison of GLUT1 expression using HSCORE between groups of RCCs based on their histological type or nuclear grade.

\begin{tabular}{lcc}
\hline Group & HSCORE & P-value \\
\hline ccRCC $^{\mathrm{b}}$ & $2.44(2.06-2.61)$ & $0.044^{\mathrm{a}}$ \\
Non-ccRCC $^{\mathrm{b}}$ & $2.01(2.00-2.03)$ & \\
ccRCC $^{\mathrm{c}}$ & $2.40 \pm 0.35$ & $0.021^{\mathrm{a}}$ \\
pRCC $^{\mathrm{c}}$ & $2.03 \pm 0.04$ & \\
ccRCC $^{\mathrm{c}}$ & $2.40 \pm 0.35$ & $0.023^{\mathrm{a}}$ \\
chRCC $^{\mathrm{c}}$ & $2.04 \pm 0.08$ & \\
pRCC $^{\mathrm{b}}$ & $2.02(2.01-2.03)$ & 0.285 \\
chRCC $^{\mathrm{b}}$ & $2.00(2.00-2.04)$ & \\
Low grade RCC $^{\mathrm{b}}$ & $2.02(2.00-2.28)$ & 0.557 \\
High grade RCC $^{\mathrm{b}}$ & $2.12(2.00-2.37)$ & \\
\hline
\end{tabular}

${ }^{\mathrm{a}} \mathrm{P}<0.05$. ${ }^{\mathrm{b}} \mathrm{Median}$ (interquartile range) and analyzed using a Mann-Whitney $\mathrm{U}$ test; ${ }^{\mathrm{c}}$ Mean \pm standard deviation and analyzed using an independant samples t-test. RCC, renal cell carcinoma; ccRCC, clear cell RCC; pRCC, papillary RCC; chRCC, chromophobe RCC.

ccRCC group exhibiting higher expression (Table II). Even after assessing the non-cc group separately by types, there was still a statistically significant difference in GLUT1 expression between ccRCCs and pRCCs $(\mathrm{P}=0.021)$ or chRCCs $(\mathrm{P}=0.023)$, again with the cc group exhibiting higher expression (Table II). There was no statistically significant difference in GLUT1 expression between pRCCs and chRCCs, or between type I and II pRCCs. To compare GLUT1 expression between RCCs of different grades, the tissues were separated into two groups, low-grade (containing nuclear grades 1 and 2) and high-grade (containing grades 3 and 4). When comparing RCCs of different nuclear grades, there was no statistically significant difference in age, sex, tumor size or GLUT1 expression between the groups (Table II). Even after separating the groups into individual grades (1-4), there was still no statistically significant difference in any of the characteristics. There was 
a weak correlation between GLUT1 expression and tumor size $(\mathrm{r}=0.33)$, however, it was not statistically significant $(\mathrm{P}=0.168)$.

\section{Discussion}

The results of the present study showed higher expression of GLUT1 in ccRCC tissues compared with pRCC and chRCC tissues. This aligns with the fact that most ccRCCs have a homozygous loss of the VHL gene, which leads to the stabilization of HIFs, increased GLUT1 mRNA transcription and ultimately, increased GLUT1 protein expression $(6,9)$. Other studies have already demonstrated higher expression of GLUT1 in ccRCCs; however, considering that there is no consensus in the literature on the quantification of the expression levels of GLUT1, these studies have used a variety of quantification methods, such as the German immunoreactive score (33-35). Certain studies have also attempted to correlate increased GLUT1 expression with clinical characteristics of RCCs, such as tumor size, tumor stage, nuclear grade and overall prognosis. The results of those studies are not consistent when taking into account certain clinical parameters, such as tumor stage and prognosis (34-37). Whilst most studies have not found a statistically significant correlation between GLUT1 expression and tumor nuclear grade or prognosis, Ambrosetti et al (34) determined that there was a statistically significant positive correlation between increased GLUT1 expression with both higher nuclear grade tumors and worse overall prognosis in ccRCCs. Furthermore, Lidgren et al (37) found a statistically significant correlation between increased GLUT1 expression and higher tumor stage at diagnosis, but only for pRCC. In the present study, a statistically significant difference between GLUT1 expression in RCCs of different nuclear grades was not found, nor was a correlation between GLUT1 expression and tumor size, in agreement with most of the referenced studies (35-37). The greater size of ccRCCs compared to non-ccRCCs corresponds to the fact that ccRCCs are more likely to be diagnosed in the first instance at a more advanced stage than the other two types of RCC assessed (7). Taking into consideration that other studies on GLUT1 expression in RCCs have used varying methods to interpret immunohistochemical expression, whilst obtaining contrasting results, the HSCORE method was used in the present study. To the best of our knowledge, no other study has used the HSCORE method for the interpretation of GLUT1 expression in RCCs, making the present study unique. An advantage that this method has over other methods of interpretation of immunohistochemical expression is the manner of data quantification. Namely, methods used in other studies quantify the proportion of positive cells in such a manner that a range of proportions is given a specific value. For example, $0-20 \%$ of positive cells in a sample is categorized with the value of $1,21-40 \%$ categorized with the value of 2 , and so on. The HSCORE method, on the contrary, takes the specific proportion of positive cells and uses that exact value to calculate the final score $(32,34-36)$. Variables that describe immunohistochemical expression are usually ordinal or discrete numerical variables for the majority of semi-quantitative methods, but in the case of the HSCORE method, continuous numerical variables are used. Continuous variables allow for more precise statistical analysis and interpretation of data. Additionally, fields of view were chosen in such a manner that a representative sample of the entire tumor was examined, whilst other studies specifically chose those fields of view that had the highest intensity of immunohistochemical expression and analyzed them (36). Another advantage of the present study was that there was no statistically significant between-group differences according to a patients sex or age at the time of diagnosis, and tumor nuclear grade, reducing potential confounding factors in the interpretation of the results. However, using immunohistochemistry as a semi-quantitative method of evaluation that is liable to the investigator's subjectivity was the main drawback of the present study. Other substantial caveats of the present study include the relatively small sample size and the fact that it was conducted in only one center. Also, no conclusions or causal relationships can be drawn due to the cross-sectional design of the study. In the future, larger, multicenter studies should be performed to increase the validity of the results. Additionally, other methods besides immunohistochemistry, such as western blotting or PCR, should be used to confirm expression.

The increased expression of GLUT1 is associated with a worse prognosis for several different types of solid tumors, which makes GLUT1 expression one of the key prognostic factors for those tumors (38); concerning RCC, most studies have not demonstrated this connection; however, it is hypothesized that the relationship is indirectly present (35-37). It is a fact that most RCCs are of the cc type, and that ccRCCs have a worse overall prognosis than other common histological types (6). It is also known that ccRCCs, unlike other histological types, exhibit increased expression of GLUT1 due to the genetic changes present in most ccRCCs $(6,9,33)$. Taking these facts into account, it can be assumed that the increased expression of GLUT1 may participate in the worse prognosis of patients with ccRCC compared with other histological types, which also coincides with studies that have demonstrated that glucose uptake is the limiting factor for further tumor growth $(39,40)$. Other than enabling enough glucose uptake for aerobic glycolysis, which maintains metabolic activity in tumor cells, the increased GLUT1 expression in RCCs is associated with reduced $\mathrm{CD}^{+}$lymphocyte infiltration of tumor tissues, which contributes to tumor tissue survival (40). The majority of studies attempting to identify the connection between GLUT1 expression and RCC prognosis observe an association either in ccRCCs alone, or in all RCC cases regardless of the histological type, most of which are ccRCC (34-37). Considering that most RCCs, wherein the majority of cases are ccRCC, exhibit increased GLUT1 expression, the differences in prognosis between individual patients likely depends on other factors, and no correlation between GLUT1 expression and prognosis has been found, to the best of our knowledge. The differences in GLUT1 expression between ccRCC cases that have the same genetic mutation, loss of VHL, can be explained by, amongst other things, different polymorphisms of the GLUT1 gene, which is supported by the fact that some GLUT1 polymorphisms have a protective role in carcinogenesis (41). GLUT1 is not studied just as a prognostic factor, but also as a predictive factor. Multiple studies have assessed the effects of GLUT1 inhibitors on cancer cells $(26-29,42,43)$. Some studies have specifically assessed the value of GLUT1 as a therapeutic target for RCC $(44,45)$. Even though GLUT1 inhibitors have 
been proven to be ineffective as a monotherapy, newer generations of anti-GLUT1 drugs show promising results when combined with conventional therapy, increasing the therapeutic benefits and lowering side effects (43). Considering that RCC is resistant to chemotherapy and radiotherapy, the treatment regimens for this metastatic disease includes immunotherapy and/or targeted therapy (5). Additionally, the official guidelines from the National Comprehensive Cancer Network even allow RCC treatment with drugs currently in clinical trials in certain cases (5). In the case of cancer progression, after all lines of therapy have been exhausted, a combination of a GLUT1 inhibitor and a conventional drug can be used as salvation therapy, if an increased expression of GLUT1 was previously proven in the same cancer, either by immunohistochemistry or by other methods.

In conclusion, the present study confirmed reports from previous studies that GLUT1 expression is increased in ccRCC compared with pRCC and chRCC. It also confirmed that GLUT1 expression does not correlate with tumor nuclear grade or tumor size. Future studies should include a larger sample size of RCCs and focus on non-clear cell RCCs with the intention to correlate GLUT1 expression with prognosis as most ccRCCs have an inherently increased expression of GLUT1, due to the genetic background of the tumor. Even though GLUT1 expression cannot be used as a prognostic marker, it might be used as a predictive marker, if future studies confirm the efficacy of anti-GLUT1 treatment on RCCs.

\section{Acknowledgements}

We would like to thank Professor Merica Glavina Durdov (Institute for Pathology, University Hospital Split) for her assistance in obtaining the antibodies needed for this research and Assistant Professor Shelly Pranić (School of Medicine, University of Split) for her expertise in language editing.

\section{Funding}

No funding was received.

\section{Availability of data and materials}

The datasets used and/or analyzed during the present study are available from the corresponding author on reasonable request.

\section{Authors' contributions}

MO, AS and SZT contributed to the conception and design of the present study. MO performed the data collection and analysis. MO, AS and SZT interpretated the results. MO drafted the manuscript. MO, AS and SZT revised the manuscript. All authors have read and approved the final manuscript. MO and SZT confirm the authenticity of all the raw data.

\section{Ethical approval and consent to participate}

The present study was approved by the Hospital Ethics Committee of the University Hospital Centre in Split, Croatia (approval no. 2181-147-01/06/M.S.-20-9), and was performed in accordance with the ethical standards described in the 1964 Declaration of Helsinki and its later amendments. Consent from patients was not required as the study was retrospective, and patients had provided consent for use of their data/samples during their visits to the hospital. Specifically, considering that the hospital is a university hospital, all patients were informed prior to surgical and/or diagnostic procedures that the material obtained during these procedures may be used for education and/or research purposes, and they signed consent forms for these procedures and the use of their data/materials.

\section{Patient consent for publication}

Not applicable.

\section{Competing interests}

The authors declare that they have no competing interests.

\section{References}

1. Padala SA, Barsouk A, Thandra KC, Saginala K, Mohammed A Vakiti A, Rawla P and Barsouk A: Epidemiology of renal cell carcinoma. World J Oncol 11: 79-87, 2020.

2. Bray F, Ferlay J, Soerjomataram I, Siegel RL, Torre LA and Jemal A: Global cancer statistics 2018: GLOBOCAN estimates of incidence and mortality worldwide for 36 cancers in 185 countries. CA Cancer J Clin 68: 394-424, 2018.

3. Srigley JR, Amin MB, Delahunt B, Campbell SC, Chang A, Grignon DJ, Humphrey PA, Leibovich BC, Montironi R, Renshaw AA, et al: Protocol for the examination of specimens from patients with invasive carcinoma of renal tubular origin. Arch Pathol Lab Med 134: e25-e30, 2010.

4. Moch H, Cubilla AL, Humphrey PA, Reuter VE and Ulbright TM: The 2016 WHO classification of tumours of the urinary system and male genital organs-part A: Renal, penile, and testicular tumours. Eur Urol 70: 93-105, 2016.

5. Motzer RJ, Jonasch E, Agarwal N, Bhayani S, Bro WP, Chang SS, Choueiri TK, Costello BA, Derweesh IH, Fishman M, et al: Kidney cancer, version 2.2017, NCCN clinical practice guidelines in oncology. J Natl Compr Canc Netw 15: 804-834, 2017.

6. Warren AY and Harrison D: WHO/ISUP classification, grading and pathological staging of renal cell carcinoma: Standards and controversies. World J Urol 36: 1913-1926, 2018.

7. Leibovich BC, Lohse CM, Crispen PL, Boorjian SA, Thompson RH, Blute ML and Cheville JC: Histological subtype is an independent predictor of outcome for patients with renal cell carcinoma. J Urol 183: 1309-1315, 2010.

8. Nickerson ML, Jaeger E, Shi Y, Durocher JA, Mahurkar S, Zaridze D, Matveev V, Janout V, Kollarova H, Bencko V, et al: Improved identification of von Hippel-Lindau gene alterations in clear cell renal tumors. Clin Cancer Res 14: 4726-4734, 2008.

9. Sadri N and Zhang PJ: Hypoxia-inducible factors: Mediators of cancer progression; prognostic and therapeutic targets in soft tissue sarcomas. Cancers (Basel) 5: 320-333, 2013.

10. Keith B, Johnson RS and Simon MC: HIF1 $\alpha$ and HIF2 $\alpha$ : Sibling rivalry in hypoxic tumour growth and progression. Nat Rev Cancer 12: 9-22, 2011.

11. Semenza GL: Hypoxia-inducible factors: Mediators of cancer progression and targets for cancer therapy. Trends Pharmacol Sci 33: 207-214, 2012.

12. Mueckler M and Thorens B: The SLC2 (GLUT) family of membrane transporters. Mol Aspects Med 34: 121-138, 2013.

13. Barron CC, Bilan PJ, Tsakiridis T and Tsiani E: Facilitative glucose transporters: Implications for cancer detection, prognosis and treatment. Metabolism 65: 124-139, 2016.

14. Hatanaka M: Transport of sugars in tumor cell membranes. Biochim Biophys Acta 355: 77-104, 1974.

15. Navale AM and Paranjape AN: Glucose transporters: Physiological and pathological roles. Biophys Rev 8: 5-9, 2016. 
16. Gu J, Yamamoto H, Fukunaga H, Danno K, Takemasa I, Ikeda M, Tatsumi M, Sekimoto M, Hatazawa J, Nishimura T and Monden M: Correlation of GLUT-1 overexpression, tumor size, and depth of invasion with 18F-2-fluoro-2-deoxy-D-glucose uptake by positron emission tomography in colorectal cancer. Dig Dis Sci 51: 2198-2205, 2006.

17. Kurokawa T, Yoshida Y, Kawahara K, Tsuchida T, Okazawa H, Fujibayashi Y, Yonekura Y and Kotsuji F: Expression of GLUT-1 glucose transfer, cellular proliferation activity and grade of tumor correlate with [F-18]-fluorodeoxyglucose uptake by positron emission tomography in epithelial tumors of the ovary. Int J Cancer 109: 926-932, 2004.

18. Park SG, Lee JH, Lee WA and Han KM: Biologic correlation between glucose transporters, hexokinase-II, Ki-67 and FDG uptake in malignant melanoma. Nucl Med Biol 39: 1167-1172, 2012.

19. Sung JY, Kim GY, Lim SJ, Park YK and Kim YW: Expression of the GLUT1 glucose transporter and p53 in carcinomas of the pancreatobiliary tract. Pathol Res Pract 206: 24-29, 2010.

20. Haber RS, Rathan A, Weiser KR, Pritsker A, Itzkowitz SH, Bodian C, Slater G, Weiss A and Burstein DE: GLUT1 glucose transporter expression in colorectal carcinoma: A marker for poor prognosis. Cancer 83: 34-40, 1998

21. Cantuaria G, Fagotti A, Ferrandina G, Magalhaes A, Nadji M, Angioli R, Penalver M, Mancuso S and Scambia G: GLUT-1 expression in ovarian carcinoma: Association with survival and response to chemotherapy. Cancer 92: 1144-1150, 2001.

22. Hoskin PJ, Sibtain A, Daley FM and Wilson GD: GLUT1 and CAIX as intrinsic markers of hypoxia in bladder cancer: Relationship with vascularity and proliferation as predictors of outcome of ARCON. Br J Cancer 89: 1290-1297, 2003.

23. Tohma T, Okazumi S, Makino H, Cho A, Mochizuki R, Shuto K, Kudo H, Matsubara K, Gunji H, Matsubara H and Ochiai T: Overexpression of glucose transporter 1 in esophageal squamous cell carcinomas: A marker for poor prognosis. Dis Esophagus 18: 185-189, 2005.

24. Yeluri S, Madhok B, Prasad KR, Quirke P and Jayne DG: Cancer's craving for sugar: An opportunity for clinical exploitation. J Cancer Res Clin Oncol 135: 867-877, 2009.

25. Hardie DG and Ashford ML: AMPK: Regulating energy balance at the cellular and whole body levels. Physiology (Bethesda) 29: 99-107, 2014

26. Melstrom LG, Salabat MR, Ding XZ, Milam BM, Strouch M, Pelling JC and Bentrem DJ: Apigenin inhibits the GLUT-1 glucose transporter and the phosphoinositide 3-kinase/Akt pathway in human pancreatic cancer cells. Pancreas 37: 426-431, 2008.

27. Wood TE, Dalili S, Simpson CD, Hurren R, Mao X, Saiz FS, Gronda M, Eberhard Y, Minden MD, Bilan PJ, et al: A novel inhibitor of glucose uptake sensitizes cells to FAS-induced cell death. Mol Cancer Ther 7: 3546-3555, 2008.

28. Zhang D, Wang Y, Dong L, Huang Y, Yuan J, Ben W, Yang Y, Ning N, Lu M and Guan Y: Therapeutic role of EF24 targeting glucose transporter 1-mediated metabolism and metastasis in ovarian cancer cells. Cancer Sci 104: 1690-1696, 2013.

29. Rastogi S, Banerjee S, Chellappan S and Simon GR: Glut-1 antibodies induce growth arrest and apoptosis in human cancer cell lines. Cancer Lett 257: 244-251, 2007.
30. Wang YD, Li SJ and Liao JX: Inhibition of glucose transporter 1 (GLUT1) chemosensitized head and neck cancer cells to cisplatin. Technol Cancer Res Treat 12: 525-535, 2013.

31. World Medical Association: World medical association declaration of Helsinki: Ethical principles for medical research involving human subjects. JAMA 310: 2191-2194, 2013.

32. Tomas SZ, Prusac IK, Roje D and Tadin I: Trophoblast apoptosis in placentas from pregnancies complicated by preeclampsia. Gynecol Obstet Invest 71: 250-255, 2011.

33. Nagase Y, Takata K, Moriyama N, Aso Y, Murakami T and Hirano H: Immunohistochemical localization of glucose transporters in human renal cell carcinoma. J Urol 153: 798-801, 1995.

34. Ambrosetti D, Dufies M, Dadone B, Durand M, Borchiellini D, Amiel J, Pouyssegur J, Rioux-Leclercq N, Pages G, Burel-Vandenbos F and Mazure NM: The two glycolytic markers GLUT1 and MCT1 correlate with tumor grade and survival in clear-cell renal cell carcinoma. PLoS One 13: e0193477, 2018.

35. Ozcan A, Shen SS, Zhai QJ and Truong LD: Expression of GLUT1 in primary renal tumors: Morphologic and biologic implications. Am J Clin Pathol 128: 245-254, 2007.

36. Kobayashi M, Uematsu T, Tokura Y, Takei K, Sakamoto K, Narimatsu T, Nukui A and Kamai T: Immunohistochemical expressionof sodium-dependent glucose transporter-2 (SGLT-2) in clear cell renal carcinoma: Possible prognostic implications. Int Braz J Urol 45: 169-178, 2019.

37. Lidgren A, Bergh A, Grankvist K, Rasmuson T and Ljungberg B: Glucose transporter-1 expression in renal cell carcinoma and its correlation with hypoxia inducible factor-1 alpha. BJU Int 101: 480-484, 2008

38. Wang J, Ye C, Chen C, Xiong H, Xie B, Zhou J, Chen Y, Zheng S and Wang L: Glucose transporter GLUT1 expression and clinical outcome in solid tumors: A systematic review and meta-analysis. Oncotarget 8: 16875-16886, 2017.

39. Singer K, Kastenberger M, Gottfried E, Hammerschmied CG, Büttner M, Aigner M, Seliger B, Walter B, Schlösser H, Hartmann A, et al: Warburg phenotype in renal cell carcinoma: High expression of glucose-transporter 1 (GLUT-1) correlates with low CD8(+) T-cell infiltration in the tumor. Int J Cancer 128: 2085-2095, 2011

40. Adekola K, Rosen ST and Shanmugam M: Glucose transporters in cancer metabolism. Curr Opin Oncol 24: 650-654, 2012.

41. Page T, Hodgkinson AD, Ollerenshaw M, Hammonds JC and Demaine AG: Glucose transporter polymorphisms are associated with clear-cell renal carcinoma. Cancer Genet Cytogenet 163: $151-155,2005$.

42. Vera JC, Reyes AM, Velasquez FV, Rivas CI, Zhang RH, Strobel P, Slebe JC, Núñez-Alarcón J and Golde DW: Direct inhibition of the hexose transporter GLUT1 by tyrosine kinase inhibitors. Biochemistry 40: 777-790, 2001.

43. Zambrano A, Molt M, Uribe E and Salas M: Glut 1 in cancer cells and the inhibitory action of resveratrol as a potential therapeutic strategy. Int J Mol 20: 3374, 2019.

44. Chan DA, Sutphin PD, Nguyen P, Turcotte S, Lai EW, Banh A, Reynolds GE, Chi JT, Wu J, Solow-Cordero DE, et al: Targeting GLUT1 and the Warburg effect in renal cell carcinoma by chemical synthetic lethality. Sci Transl Med 3: 94ra70, 2011.

45. Shuch B, Linehan WM and Srinivasan R: Aerobic glycolysis: A novel target in kidney cancer. Expert Rev Anticancer Ther 13: 711-719, 2013. 